\title{
Molecular aspects of primary immunodeficiencies: lessons from cytokine and other signaling pathways
}

\author{
Fabio Candotti, ${ }^{1}$ Luigi Notarangelo, ${ }^{2}$ Roberta Visconti, ${ }^{3}$ and John O’Shea ${ }^{4}$ \\ ${ }^{1}$ Genetics and Molecular Biology Branch, National Human Genome Research Institute, NIH, Bethesda, Maryland, USA \\ ${ }^{2}$ Department of Pediatrics, University of Brescia, Brescia, Italy \\ ${ }^{3}$ Department of Pathology, University Frederico II, Naples, Italy \\ ${ }^{4}$ Molecular Immunology and Inflammation Branch, National Institute of Arthritis and Musculoskeletal and Skin Diseases, \\ $\mathrm{NIH}$, Bethesda, Maryland, USA
}

Address correspondence to: John O'Shea, National Institute of Arthritis and Musculoskeletal and Skin Diseases, NIH, 10 Center Drive, Building 10, Room 9N262, Bethesda, Maryland 20892-1820, USA.

Phone: (301) 496-6026; Fax: (301) 402-0012; E-mail: osheajo@mail.nih.gov.

J. Clin. Invest. 109:1261-1269 (2002). doi:10.1172/JCI200215769.

Numerous examples indicate that mammalian development can be entirely normal in the absence of the immune system; indeed, prior to birth, individuals with even very severe immunodeficiencies are developmentally unaffected. Perhaps it is not surprising, therefore, that more than 95 different primary immunodeficiency syndromes have been identified, encompassing defects in lymphocytes, phagocytes, and complement proteins. Indeed, within the past several years, more than 70 separate genes have been identified whose mutations cause immunodeficiency. These discoveries have been made both by using candidate gene approaches and by positional cloning. In some cases, the generation of gene-targeted mice preceded the identification of human mutations, whereas in other cases, the reverse was true. Therapy for these disorders, ranging from replacement therapy to bone marrow transplantation and gene therapy, has also moved at a rapid pace. This field therefore provides outstanding examples of the power of molecular medicine, with tremendous opportunities for interplay between basic and clinical science.

Many of the processes that govern development of lymphoid and hematopoietic cells are now understood in some detail. We know that the growth and development of hematopoietic precursors, which develop in the fetal liver and bone marrow, are dependent upon a panoply of cytokines. In addition, lymphocytes require appropriate signals from antigen receptors to mature properly (Figure 1). Additionally, other receptors and counter-receptors on lymphoid and antigen-presenting cells are critical for initiating immune responses (Figure 2). We will provide examples in which mutations affect each of these steps and consequently result in immunodeficiency (Table 1). Several excellent reviews provide comprehensive discussion of the genetic basis of primary immunodeficiencies $(1,2)$. Our goal is not to summarize this field in its entirety; rather, a major focus of this review will be the role of cytokines and their receptors in the pathogenesis of primary immunodeficiencies. Additionally, the identification of new genes associated with immunodeficiency disorders, insights from patient-derived mutations, the heterogeneity of clinical presentations, the significance of revertants, and advances in gene therapy will be highlighted. To an extent, the areas emphasized are also a reflection of our interests, but in general the lessons are applicable to most of the diseases encompassed by primary immunodeficiencies.

\section{Disorders of cytokines and cytokine receptors}

Cytokines and receptors of different classes are important in lymphoid development and function. We will briefly consider cytokines that bind the common $\gamma$ chain, $\gamma_{c}$; cytokines involved in cell-mediated immunity; and members of the TNF superfamily.

\section{$\gamma_{\mathrm{c}}$ cytokines, Jak3, and lymphocyte development}

IL-2, IL-4, IL-7, IL-9, IL-15, and IL- 21 bind $\gamma_{c}$ in association with a unique ligand-specific receptor chain. The involvement in signaling via multiple cytokines and its mapping to the $\mathrm{X}$-chromosome led to the identification of mutations of $\gamma_{c}$ in boys with $\mathrm{X}$-linked severe combined immunodeficiency (SCID). The disease is now termed SCIDX1 and is the most common type of SCID, accounting for about $50 \%$ of cases $(2,3)$. Through the generation of knockout mice, one $\gamma_{c}$ cytokine, IL-7, was shown to be an essential cytokine for $\mathrm{T}$ cell development; SCID patients with IL-7 receptor (IL-7R) mutations were subsequently identified (4). However, absence of $\gamma_{c}$ interferes with both IL-7 and IL-15 signaling; loss of IL-15 or its receptor abrogates NK cell development. Thus, IL-7R deficiency is associated with absence of T cells and residual NK cells, whereas $\gamma_{c}$ deficiency typically results in absence of both lymphoid subsets. 
Intracellularly, these receptor subunits associate with the Janus kinases Jak1 and Jak3. Jak1 is widely expressed and is used by many different cytokine receptors, and its knockout is perinatally lethal. In contrast, Jak3 is predominantly expressed in hematopoietic cells and uniquely binds $\gamma_{c}$. Consequently, JAK3 deficiency, the second most common cause of $\mathrm{T}^{-} \mathrm{B}^{+} \mathrm{SCID}$, has a phenotype identical to that of $\operatorname{SCIDX} 1(5,6)$. More than 30 patients with more than 35 different mutations spanning the entire JAK3 gene have been identified (7). These mutations are stochastic, with no evidence of founder effects or hot spots. About half of the patients are compound heterozygotes, whereas half are homozygous for their mutations, due to parental consanguinity. These mutations appear to be fully recessive in that the level of JAK3 protein is normal in heterozygotes; the factors that control JAK3 expression are presently being investigated and likely include both transcriptional and posttranscriptional mechanisms.

Most mutant JAK3 alleles fail to encode any stable protein, but several missense or small in-frame deletion alleles have been identified that permit near-normal levels of expression of the mutant gene product. The analysis of the effect of these mutations on JAK3 function has greatly improved our understanding of how this tyrosine kinase works. Jaks comprise a C-terminal catalytic domain, preceded by a unique "pseudokinase" domain, which is a frequent site of mutations. Analysis of these mutant alleles has demonstrated the critical function of this domain (8). The pseudokinase domain is not catalytically active but instead has important regulatory functions, perhaps through binding to the kinase domain. The N-terminus of the Jaks has homology to a conserved domain in band four point one, ezrin, radixin, and moesin (FERM). Mutations in the JAK3 FERM domain have two important consequences: they disrupt binding to $\gamma_{c}$, and they interfere with catalytic activity (9).

The clinical presentations associated with JAK3 mutations are surprisingly variable, with about onethird of patients developing T cells. Similarly, a missense mutation of $\gamma_{c}$ has been associated with normal numbers of peripheral $\mathrm{T}$ and $\mathrm{B}$ cells, grossly normal $\mathrm{T}$ cell receptor repertoire, normal response to mitogenic stimuli, and the presence of a normal thymus (10). Taken together with the identification a kindred with a different missense mutation of $\gamma_{c}$ that exhibited attenuated immunodeficiency (11), this indicates that clinical presentations associated with JAK3 and $\gamma_{c}$ mutations can be remarkably broad.

Table 1

Defects leading to primary immune deficiency

\begin{tabular}{|c|c|c|}
\hline Class of defect & Gene & Disease \\
\hline $\begin{array}{l}\text { Disorders of cytokines and } \\
\text { cytokine signaling }\end{array}$ & $\begin{array}{l}\gamma_{c} \text {, Jak3, IL-7R } \\
\text { IL-2R } \alpha \\
\text { IFN- } \gamma \text { R1, IFN- } \gamma \text { R2, IL-12p40, IL-12R } \beta 1 \text {, Stat1 } \\
\text { TNFR1 } \\
\text { Fas, caspase-10 } \\
\text { CD40, CD40L, AID } \\
\text { IKK } \gamma \\
\text { FOXP3 }\end{array}$ & $\begin{array}{l}\text { T-B }{ }^{+} \text {SCID } \\
\text { Lymphoproliferative disease } \\
\text { Atypical mycobacterial infection } \\
\text { TRAPS } \\
\text { ALPS } \\
\text { HIGM } \\
\text { Hypohidrotic ectodermal dysplasia } \\
\text { IPEX }\end{array}$ \\
\hline
\end{tabular}

Antigen presentation, receptors, and signaling

Accessory and adhesion molecules

Metabolic

Tap1, Tap2
RFXAP, CIITA, RFX5, RFXANK
Rag1, Rag2
Artemis
DNA ligase IV
Nijmegen breakage syndrome
ATM
Ig $\alpha, \lambda 5, \mu$-chain
BLNK
Btk
CD3 $\gamma$, CD3e,
Lck
Zap70
CD45
WASP
CD18 ( $\beta 2$ integrin)
GDP-fucose transporter
SH2D1A
ADA
PNP

Impaired $\mathrm{MHC}$ class I expression and $\mathrm{CD}^{+} \mathrm{T}$ cell development Impaired $\mathrm{MHC}$ class II expression and $\mathrm{CD} 4^{+} \mathrm{T}$ cell development SCID

SCID with radiosensitivity

SCID

Immunodeficiency

Ataxia-telangiectasia

Agammaglobulinemia

Agammaglobulinemia

X-linked agammaglobulinemia

T cell deficiency

SCID

$\mathrm{CD}^{+} \mathrm{T}$ cell deficiency

SCID

WAS

LAD I

LAD II

XLP

SCID

CID 


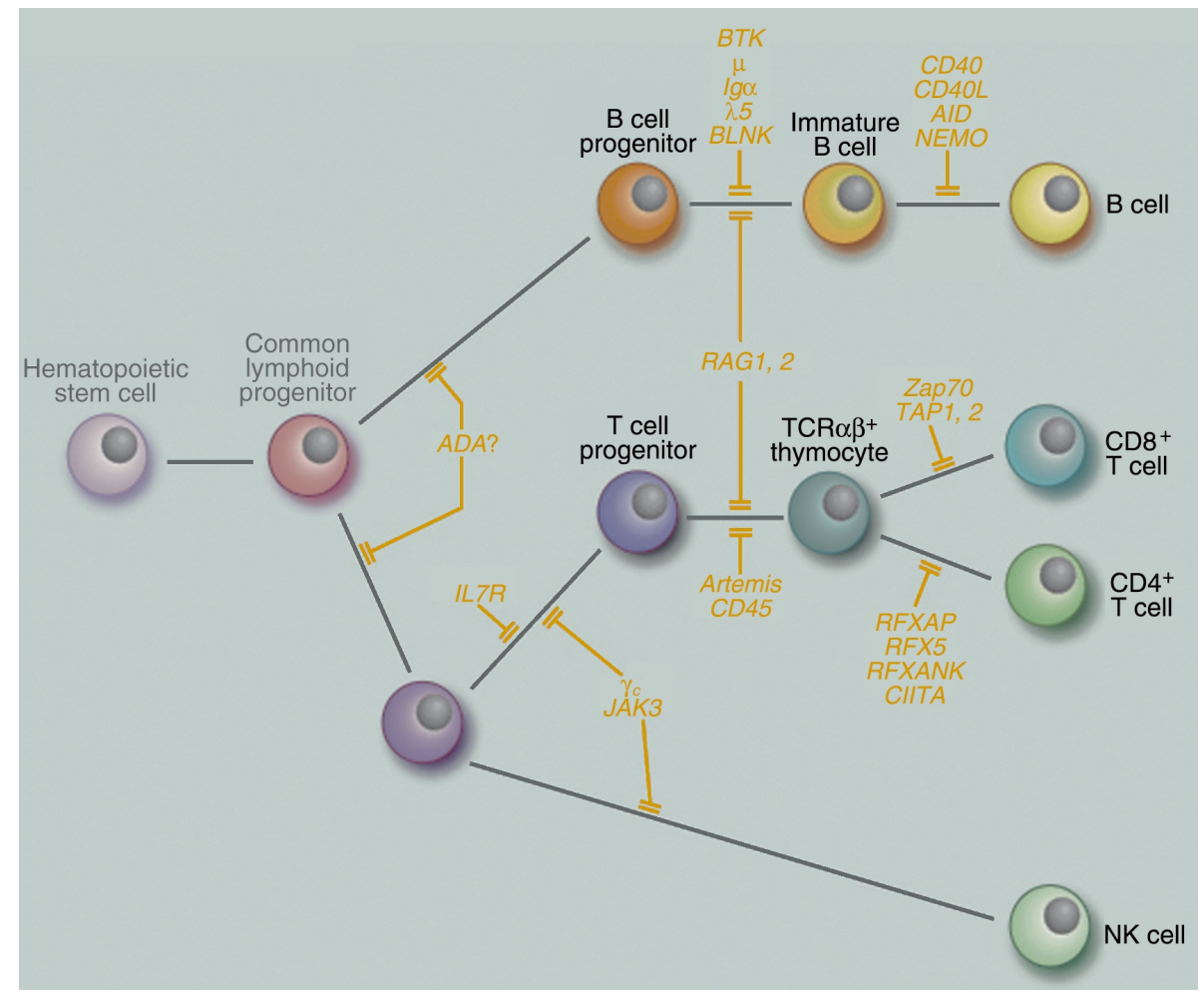

Figure 1

Schematic representation of lymphoid development and genetic lesions leading to immunodeficiency.

Lack of $\gamma_{c}$ or Jak3 interferes with signaling by not only IL-7 and IL-15 but also IL-2, a cytokine that acts in vivo to constrain lymphoid growth by promoting activationinduced cell death and maintaining peripheral tolerance. These effects are best illustrated in humans and mice with a lymphoproliferative disease resulting from mutations in the IL-2R $\alpha$ and IL-2R $\beta$ subunits (12). Recently, we described a SCID kindred with mutations in JAK3. One child in this family developed severe lymphoproliferative disease, but curiously, a sibling was clinically nearly normal but had lymphopenia with oligoclonal $\mathrm{T}$ cell expansion and increased numbers of activated and memory $\mathrm{T}$ cells. These cells also had poor expression of the proapoptotic molecule Fas ligand, which is typically upregulated by IL-2. Indeed, the T cells that develop in $\gamma_{c}$-and Jak3-deficient mice and humans often express activation markers (13).

Thus, a feature of JAK3 deficiency appears to be impaired lymphoid homeostasis, further complicating the clinical phenotype. This disorder can range from pure immunodeficiency to a mixed picture with varying degrees of autoimmunity and immunodeficiency and, in some cases, minimal clinical consequences. Indeed, it is becoming increasingly clear that $\mathrm{T}^{-} \mathrm{B}^{+}$SCID represents just one extreme of a range of clinical presentations associated with Jak 3 and $\gamma_{c}$ mutations. There are other examples of syndromes with a mixed picture of autoimmune and immunodeficiency disease - immune dysregulation polyendocrinopathy X-link and syndrome (IPEX) and autoimmune polyendocrinopathy-candidiasis-ectodermal dystrophy (APECED) being examples. The former is due to mutations of the FOXP3 gene, which encodes a putative winged-helix/forkhead transcription factor and is thought to serve as a transcriptional repressor of cytokine genes (14). APECED is associated with mutations of the AIRE (autoimmune regulator) gene, which is also thought to be a transcriptional regulator $(15,16)$.

\section{IL-12 and IFN- $\gamma$ : cytokines that regulate Th1 differentiation and cell-mediated immunity}

After development in the thymus, naive $\mathrm{CD} 4^{+}$cells differentiate to Th1 cells that produce IFN- $\gamma$, a cytokine that promotes cell-mediated immunity; IFN- $\gamma$ activates the transcription factor Stat1 (signal transducer and activator of transcription-1). One cytokine that regulates this process, IL-12, is composed of two subunits, IL-12p 40 and IL-12p35. Its receptor is also a heterodimer, consisting of IL-12R $\beta 1$ and IL-12R $\beta 2$. Patients with mutations in the genes for either IFN- $\gamma$ receptor subunit (IFNGR1 or IFNGR2), or for STAT1, IL-12p 40, or IL-12R $\beta 1$, present with atypical mycobacterial and Salmonella infections $(17,18)$. Mice with IFN- $\gamma$, IFN- $\gamma$ R, and Stat 1 deficiencies show increased incidence of tumors, but whether human mutations of these constituents are associated with increased risk of cancer remains to be determined.

\section{The TNF and TNF receptor superfamilies}

Another large family of cytokine receptors, with more than 20 members that bind both soluble and cellbound ligands, is the TNF superfamily. Mutation of 


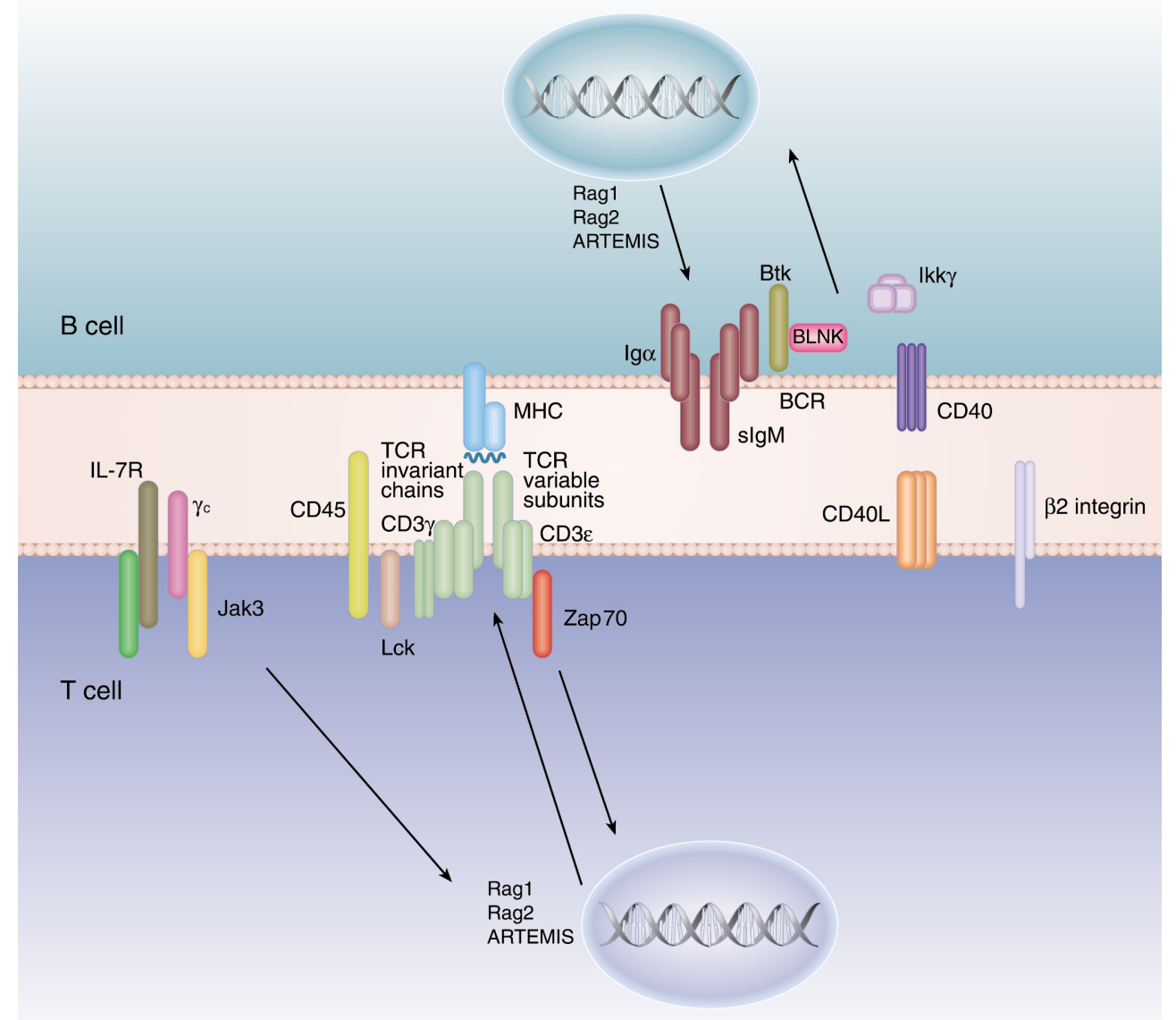

\section{Figure 2}

The development of lymphoid cells is dependent upon the expression and signaling by cytokine receptors, antigen receptors, and adhesion/accessory molecules. Mutations of many different genes can interfere with proper lymphoid development and function and, consequently, can lead to immunodeficiency.

TNF receptor I (TNFRI or TNFSFRIA) is associated with a periodic fever and autoinflammatory disease termed TNFR-associated periodic syndrome (TRAPS) (19). TRAPS is one of a group of heritable periodic fever syndromes that also includes familial Mediterranean fever, Muckle-Wells syndrome, and hyper-IgD syndrome (20). Another receptor/ligand pair in the TNFR family is Fas/FasL, which regulates lymphocyte apoptosis through the activation of intracellular caspases. Deficiency of Fas and FasL was first identified in mice, where their essential proapoptotic functions are evident. Later, humans with mutations of FAS and CASP10 (which encodes another proptotic molecule, caspase-10) were identified (21-23); these patients have autoimmune lymphoproliferative disorder and are at increased risk of developing lymphomas. In terms of immunodeficiency, the TNF-family receptor/ligand pair that is most relevant and best characterized is CD40/CD40L (CD154).

\section{CD40/CD40L and hyper-IgM syndrome}

CD40L (CD154 or TNFSF5) is expressed on activated $\mathrm{CD}^{+} \mathrm{T}$ cells, whereas its counter-receptor, $\mathrm{CD} 40$, is widely expressed on $\mathrm{B}$ cells, macrophages, dendritic cells (DCs), and other cells. Maturation of antibody responses occurs in the germinal centers (GCs) and involves the mechanisms of somatic hypermutation (SHM) and class switch recombination (CSR). Through SHM, B cells modify their rearranged Ig genes, generating diversity and high-affinity antibodies. With CSR, B lymphocytes modify their Ig constant region and produce antibodies with distinct biological properties. The cognate interaction between CD40 ligand (CD40L), expressed by activated $\mathrm{CD} 4^{+} \mathrm{T}$ cells, and CD40 on B lymphocytes provides a key signal. This is disrupted in patients with $C D 40 \mathrm{~L}$ or $C D 40$ mutations, who suffer from X-linked immunodeficiency with hyper-IgM (HIGM1) and autosomal-recessive hyperIgM, respectively $(24,25)$. HIGM1 is characterized by a lack of GCs and impaired CSR due to lack of T cell stimulation. The finding that SHM occurs normally in affected individuals suggests that SHM is not strictly dependent on CD40L-CD40 interaction and may occur outside of the GCs. Unlike HIGM1, CSR is precluded in CD40-deficient patients due to an intrinsic B cell defect. The concerted action of genes expressed by $T$ and B lymphocytes and follicular DCs oversees the GC reaction, thus exposing terminal $\mathrm{B}$ cell differentiation to the consequences of inherited gene mutations.

Signaling through CD40 involves activation of TNF receptor associated factor 6 (TRAF6), activation of the inhibitor of $\kappa B$ kinase (IKK) complex, and induction 
of NF- $\mathrm{KB}$. Mutations of the $\gamma$ chain of the IKK complex (also known as NF-KB essential modulator, or NEMO) have been shown to impair NF-KB signaling $(26,27)$. Affected patients suffer from immune deficiency with hyper-IgM Ig profile due to impaired CSR and hypohidrotic ectodermal dysplasia.

Another form of autosomal-recessive hyper-IgM (HIGM2) has been recently described in patients with mutations of activation-induced cytidine deaminase (AID), a novel enzyme that likely plays a main role in the control of both CSR and SHM (28). AID is selectively expressed in the GC and can be induced in $\mathrm{B}$ cells following stimulation with LPS or CD40L and appropriate cytokines. AID-deficient patients show recurrent infections and enlarged GCs in lymph nodes and tonsils containing abundant proliferating $\mathrm{IgM}^{+} \mathrm{IgD}^{+} \mathrm{CD} 38^{+} \mathrm{GC}$ founder $\mathrm{B}$ cells.

\section{Antigen receptors and receptor-mediated signaling} It is important to emphasize that in addition to cytokine signals, signals provided by antigen receptors represent a key event that allows lymphoid differentiation to proceed to the production of mature $\mathrm{T}$ and $\mathrm{B}$ lymphocytes. Accordingly, deficiencies of components of $\mathrm{T}$ and $\mathrm{B}$ cell antigen receptors can result in failure to develop these subsets. Multiple types of mutations can interfere with antigen receptor signaling, ranging from impairment antigen presentation and defects in the assembly of the antigen receptor complex to deficiency of signaling molecules.

\section{Defective MHC class I and class II presentation}

Most $\mathrm{T}$ cells require antigen to be presented by MHC molecules, class I for $\mathrm{CD}^{+}$cells and class II for $\mathrm{CD} 4^{+}$ cells. Mutation of TAP1 or TAP2 impairs expression of HLA class I molecules on the cell surface; as predicted, this interferes with the development of $\mathrm{CD}^{+}$but not $\mathrm{CD} 4^{+} \mathrm{T}$ cells. Patients with TAP deficiency suffer from recurrent infections and deep skin ulcers. A variety of transcription factors, including RFXAP, CIITA, RFX5, and RFXANK, control MHC class II expression, and mutation of any these leads to combined immunodeficiency. Recently a missense mutation of CIITA was identified in three sisters, one of whom was asymptomatic despite a profound reduction in class II expression (29), a finding that underscores once again the heterogeneity that can be seen in patients with primary immunodeficiencies.

\section{Defects of $V(D) J$ recombination and DNA repair}

Lymphoid antigen receptors comprise invariant subunits associated with gene products generated by the rearrangement of single variable $(\mathrm{V})$, diversity $(\mathrm{D})$, and joining $(J)$ elements with constant region genes. The invariant subunits are important for signal transduction, whereas the rearranged subunits are responsible for antigen recognition; both, however, are required for lymphoid development. A series of proteins encoded by lymphoid-restricted recombinase-activating genes 1 and 2 (RAG1 and RAG2) or ubiquitously expressed genes (DNA-PKcs, Ku70, Ku80, XRCC4, and
DNA ligase IV) mediate the recombination process. Inactivation of any of these gene products results in a block in $\mathrm{T}$ and $\mathrm{B}$ cell development and profound immune deficiency. In particular, disruption of either Rag1 or Rag2 in mice and humans causes SCID with a virtual lack of circulating mature $\mathrm{T}$ and $\mathrm{B}$ lymphocytes (T-B- SCID) (30). Interestingly, mutations that impair but do not abrogate RAG1 and RAG2 protein expression and function result in a distinct human phenotype (Omenn syndrome), characterized by a lack of circulating B cells, with presence of a substantial number of oligoclonal, activated, and poorly functioning $\mathrm{T}$ lymphocytes, mimicking graft-versus-host disease (31). In addition, background genetic or perhaps environmental factors also seem to play a role in determining the clinical phenotype of RAG mutations, as $\mathrm{T}^{-} \mathrm{B}^{-}$SCID, Omenn syndrome, and atypical forms of SCID may coexist within the same family, or in distinct families with the same mutation.

Additionally, a newly identified player, ARTEMIS, which complexes with DNA-PKcs and is thought to provide hairpin-opening activity in $\mathrm{V}(\mathrm{D}) \mathrm{J}$ recombination, has been recently linked to the clinical subgroup of patients with $\mathrm{T}^{-} \mathrm{B}^{-}$SCID associated with increased radiosensitivity $(32,33)$. Interestingly, ARTEMIS maps at $10 \mathrm{p}$, in a region where the locus for a similar SCID condition found in Athabascan-speaking Native Americans had been mapped. Whether defects in the ARTEMIS gene account for all cases of radiation-sensitive $\mathrm{T}^{-} \mathrm{B}^{-} \mathrm{SCID}$ in humans, and whether mutations that allow residual expression of the ARTEMIS protein result in a milder phenotype, remain to be established.

Although disruption of genes for DNA-PKcs, Ku70, Ku80, or XRCC 4 has been shown to cause SCID in mice, mutations of the corresponding human genes have not been identified thus far. However, DNA ligase IV, which functions in DNA nonhomologous end-joining and $V(D) J$ recombination, was recently demonstrated to be mutated in patients with features of immunodeficiency and developmental delay (34), a syndrome resembling that of the DNA damage response disorder Nijmegen breakage syndrome. Another related disorder, ataxia-telangiectasia (AT), is an autosomal-recessive condition characterized by cerebellar ataxia and progressive neuromotor disability associated with oculo-cutaneous telangiectasia, immunodeficiency of variable degree with absence or degeneration of the thymus, and high predisposition to lymphoid malignancies. AT is also associated with chromosomal instability and increased sensitivity to ionizing radiation. The gene responsible for AT is designated ATM; the predicted ATM protein has homology to mammalian phosphatidylinositol $3^{\prime}$-kinase and DNA-dependent protein kinase $(35,36)$.

\section{Mutations of invariant chains and signaling molecules}

Both $\mathrm{T}$ and $\mathrm{B}$ cell antigen receptors employ invariant chains to initiate signal transduction. For the $\mathrm{T}$ cell receptor (TCR), CD3 proteins and TCR $\zeta$ serve this function; mutations of $\mathrm{CD} 3 \gamma$ and $\mathrm{CD} 3 \varepsilon$ have been 
identified. Similarly, the invariant chains for the B cell receptor (BCR) are Ig $\alpha(\mathrm{CD} 79 \mathrm{a})$ and $\operatorname{Ig} \beta(\mathrm{CD} 79 \mathrm{~b})$, and mutation of the former has been found in some patients with agammaglobulinemia. These invariant chains are phosphorylated in response to receptor cross-linking on tyrosine residue motifs termed ITAMs (immunoreceptor-based tyrosine activation motif). In T cells, this is mediated by the protein tyrosine kinase (PTK) Lck, and phosphorylation of the receptor allows recruitment of a second kinase, Zap70; deficiency of both Lck and Zap70 is also associated with $\operatorname{SCID}(37,38)$. Zap70 deficiency is characteristic in that $\mathrm{CD} 4^{+} \mathrm{T}$ cells are produced but $\mathrm{CD} 8^{+} \mathrm{T}$ cells are absent. The tyrosine phosphatase CD45 is also a key regulator of TCR signaling. Mutation of it was recently demonstrated, and this leads to $\mathrm{T}^{-} \mathrm{B}^{+}$ SCID in humans (39).

Bruton's tyrosine kinase (BTK), a key PTK expressed in B cells, is mutated in X-linked agammaglobulinemia (XLA), a severe immunodeficiency that affects approximately 1 in 50,000 male live births (40). Typically, XLA patients manifest a block in B cell development at the pre-B cell stage and do not produce Ig's. However, the clinical presentations associated with $B T K$ mutations are quite heterogeneous. A considerable number of patients have a milder disorder in which B cells develop and variable amounts of Ig's are produced. Here again, there is no clear genotype-phenotype correlation, and a range of clinical phenotypes can be found within a given family. Roughly $10 \%$ of patients with agammaglobulinemia do not have mutations of BTK. Such patients may have mutations in the autosomal genes for Ig chains, such as the $\mu$ heavy chain or a surrogate light chain gene $(\lambda 5)$. Still others carry mutations in BLNK, an adapter molecule that contributes to BCR signaling and is required for the development of proB to pre-B cells (41).

Another X-linked disorder, Wiskott-Aldrich syndrome (WAS), is characterized by thrombocytopenia with small-sized platelets, eczema, and immune deficiency, leading to increased susceptibility to infection from all classes of pathogens and high susceptibility to lymphoid cancer. The disease gene was discovered by positional cloning (42) and encodes the WAS protein (WASP), which is expressed in all nonerythroid hematopoietic cells. Together with N-WASP and WAVE, WASP is a member of a family of human proteins responsible for the transduction of signals from the cell membrane to the actin cytoskeleton. WASP interactions with Rho family GTPases and the Arp $2 / 3$ complex are critical for this function and affected by WASP mutations, which provide an explanation for several of the biological defects characteristic of WAS hematopoietic cells, including abnormal signaling, polarization, migration, and phagocytosis (reviewed in ref. 43). Intramolecular interactions maintain WASP in an autoinhibited status that is relieved by binding of Cdc 42 to the WASP GTPasebinding domain. This event releases the C-terminal region of the protein and enables its interaction with the Arp $2 / 3$ complex, thus resulting in localized polymerization of new actin filaments (44). Interestingly, recent findings have shown that mutations of WASP disrupting the autoinhibitory domain do not result in the WAS phenotype, but in an X-linked severe congenital neutropenia (45).

\section{Accessory and adhesion molecules}

In addition to antigen receptors, lymphocytes have an array of accessory and adhesion molecules that are important for providing costimulatory signals for $\mathrm{T}$ cell activation. Some adhesion molecules are also important for the function of phagocytic cells. Mutations of CD18, a $\beta 2$ integrin, have long been known to result in the disorder leukocyte adhesion deficiency (LAD I). Additionally, a second form of leukocyte adhesion deficiency (LAD II) is caused by deficient expression of sialyl Lewis $X$ determinants, the neutrophil ligands for E- and P-selectins. Patients affected by LAD II present with less severe infections than LAD I patients, along with developmental abnormalities, short stature, and mental retardation. The transport of GDP-fucose into isolated Golgi vesicles of LAD II cells is reduced in patient cells, and a point mutation in a GDP-fucose transporter has been recently demonstrated to be responsible for the disease in one patient $(46,47)$.

The disorder $\mathrm{X}$-linked lymphoproliferative disease is characterized by fatal Epstein-Barr virus (EBV) infection, lymphomas, immunodeficiency, aplastic anemia, and lymphohistiocytic disorders. It appears to result from the failure to control the proliferation of cytotoxic T cells following EBV infection; as such, it may be considered both an autoimmune and an immunodeficiency disease. The gene underlying $\mathrm{X}$-linked lymphoproliferative syndrome, SH2D1A (SH2 domain-containing gene $1 \mathrm{~A}$ ), which is expressed in $\mathrm{T}$ and NK cells (48), encodes a small adapter protein with a single SH2 domain. SH2D1A binds SLAM (CD150), 2B4 (CD244), and perhaps other lymphoid receptors. The ligand for CD244 is CD48, which is upregulated with EBV infection. Whether SH2D1A is a positive or a negative regulator of signaling is still unclear, and the pathogenesis of this disorder is still incompletely understood. The disease has some features similar to familial hemophagocytic lymphohistiocytosis, which results from mutations of the gene encoding perforin (49), and Griscelli syndrome, which is due to RAB27A and MYO5A mutations that interfere with granule exocytosis $(50,51)$.

\section{Metabolic defects}

Genetic deficiency of adenosine deaminase (ADA) results in a clinical spectrum of immunodeficiency that ranges from infants with classical SCID to adultonset presentation with milder combined immunodeficiency (CID) phenotype (reviewed in refs. 52, 53). The molecular basis of ADA deficiency has been known for three decades; however, important biological and clinical challenges still exist that make investigation of this disease current and attractive. The 
pathogenic mechanisms responsible for immunodeficiency in ADA deficiency remain uncertain. In the purine salvage pathway, ADA catalyzes the deamination of adenosine (Ado) and deoxyadenosine (dAdo) to inosine and deoxyinosine, respectively. In the absence of the enzyme, accumulation of dAdo results in a massive increase of dATP levels in the patients' red blood cells and lymphocytes. High concentrations of dAdo and dATP have profound metabolic consequences, including chromosome breakage, activation of apoptosis, inhibition of methylation reactions and DNA synthesis, and activation of ATP catabolism, all of which are thought to mediate lymphotoxicity and account for the severe depletion of both $\mathrm{T}$ and $\mathrm{B}$ lymphocytes in affected patients $\left(\mathrm{T}^{-} \mathrm{B}^{-}\right.$ SCID). Recent studies in ADA knockout mice have shown abundant $\mathrm{T}$ cell apoptosis in the thymus (but not in the spleen and lymph nodes), accompanied by reduction of tyrosine phosphorylation of TCR-associated signaling molecules and block of TCR-mediated calcium flux. These findings suggest that, in addition to the direct apoptotic effects of dAdo and dATP, blocks in TCR-driven thymocyte maturation may play a role in the $\mathrm{T}$ cell depletion observed in ADA deficiency.

\section{Spontaneous revertants and gene therapy}

Primary immunodeficiencies fully fit Lord Garrod's 1924 definition of "Experiments of Nature" and have recently provided fascinating examples of Nature's attempts to correct its own missteps. Reversion of mutations to normal sequence has in fact been demonstrated in a series of immunodeficient patients with mild phenotype or progressive improvement of their clinical presentation. In vivo reversion to normal of an inherited mutation has been described in an ADA-deficient child with progressive clinical improvement and unusually mild biochemical and immunologic phenotype (54). A second case of in vivo reversion in ADA deficiency was recently identified in a subject with a relatively mild biochemical and immunological phenotype, who had acquired a deletion that abrogated the deleterious effects of the original mutation (55).

A reversion of a point mutation to normal sequence was also demonstrated in a boy with an attenuated form of SCIDX1 (56). In this case, the reversion appears to have occurred in a committed $\mathrm{T}$ cell progenitor, as the patient presented with low-to-normal numbers of circulating T cells expressing the normal $\gamma_{c}$ molecule, in contrast to B lymphocytes, monocytes, and granulocytes, which all carried the original point mutation and had no detectable $\gamma_{c}$ expression.

Somatic mosaicism sustained by true back mutations in $\mathrm{T}$ lymphocytes has also been described in two patients affected with WAS $(57,58)$. In both cases, WASP-positive and WASP-negative circulating T lymphocytes coexisted, but no revertants could be detected among other lymphoid or myeloid populations. In one case, the proband's condition ameliorated. This individual's peripheral $\mathrm{T}$ cells were largely of the revertant genotype, suggesting that the reversion event had resulted in clinical improvement, although this was ascertained retrospectively.

The observation of these immunodeficient patients with attenuated phenotype and carrying reversions of their original mutation adds a level of complexity to the clinical interpretation of primary immunodeficiencies as it broadens the spectrum of possible presentation. Whenever normal cells enjoy a selective advantage over cells with a mutant phenotype, reversions to the wild type can lead to substantial somatic mosaicism and relatively mild symptoms - a possibility that should be considered each time we are confronted with atypical presentations of known immunodeficiency syndromes.

Such in vivo reversions can be considered as spontaneous forms of gene therapy and can provide important information as to the legitimate chances of success of genetic correction strategies for immunodeficiency. In particular, the above-described case of somatic mosaicism due to reversion of $\gamma_{c}$ mutation had suggested that gene therapy should be an efficient form of treatment for SCIDX1, a prediction that was recently confirmed by the results of a successful clinical trial (59). In this recent report, CavazzanaCalvo and coworkers reported on the results from five patients treated with autologous $\mathrm{CD} 34^{+}$bone marrow cells in which the cDNA encoding for human $\gamma_{c}$ had been transferred using a retroviral vector. This procedure resulted in the appearance of normal numbers of circulating $T$ lymphocytes expressing $\gamma_{c}$ in four out of five patients. These $\mathrm{T}$ lymphocytes were polyclonal and functionally competent, as demonstrated by normal responses to stimulation with mitogens and specific antigens. In addition, despite the fact that less than $1 \%$ of the patients' B cells became $\gamma_{c}$-positive, the first two treated patients developed specific antibody responses to tetanus and diphtheria toxins, as well as polioviruses. Not surprisingly, based on the predicted lack of selective advantage in myeloid lineages, only $0.01-1 \%$ of the patients' monocytes and granulocytes showed evidence of genetic correction. The observation of adequate humoral immune responses in the presence of very low numbers of gene-corrected B lymphocytes may indicate that the humoral immunodeficiency observed in untreated SCIDX1 patients is due mostly to lack of $\mathrm{T}$ cell helper function and less to intrinsic deficiencies of their B lymphocytes.

These results are regarded as sensational, especially when compared with previous outcomes of hematopoietic stem gene therapy for such other immunodeficiency diseases as ADA deficiency $(60,61)$, chronic granulomatous disease (62), and LAD I (63), which failed to demonstrate clear clinical benefit. There is now high hope that the application of the same technical progress - including the use of fibronectin cell culture support, novel cytokines, and modern vector design - will lead to similar successes in gene therapy for other immunodeficiencies. Indeed, novel gene therapy approaches for ADA deficiency have generated promising preliminary results (A.S. Aiuti, personal communication). 


\section{Conclusions}

In this review, we have tried to emphasize several themes. First, since most genes that regulate immune responses are not essential for normal organogenesis and development, mutations of numerous components can be found in the population. Many more such mutations are likely to be found affecting innate and adaptive immune response. It would not be surprising at all if the present number of seventy-odd immunodeficiency-related genes doubled in the next few years. Mutations in other TNF receptors, Toll receptors, and the numerous chemokine receptors may well be found. It is intriguing to speculate how such mutations might present in humans.

Our second point is that the clinical phenotype associated with these mutations is amazingly unpredictable. While knockout mice have been useful in providing clues to the importance of a given gene, the clinical significance of the corresponding human mutations vary even within a single family. Thus, more than one gene can cause similar immunodeficiency, and a single gene can have rather variable clinical presentation. Given the surprisingly atypical presentations associated with these disorders, mutations may be far more common than we think. Improvements in sequencing technology will permit easier and more rapid analysis of larger numbers of patients. It will be of great interest to determine just how frequent mutations and polymorphisms are. It will be important as well to try to determine what modifier genes influence the severity of primary immunodeficiencies.

Third, immunodeficiency and autoimmunity are not the opposites that one might think them to be a priori; indeed, mixed pictures of autoimmunity and immunodeficiency are quite common. The more we learn about the immune system, the more apparent are the complex functions of many of its components, which often play both positive and negative roles. IL-2 is an outstanding example, but TNF and other cytokines can also be proinflammatory and immunosuppressive in different contexts. Consequently, it is not surprising that primary immunodeficiency and autoimmunity can go hand in hand. The common autoimmune diseases are not simple mendelian disorders but rather are polygenic. It is tempting to speculate that we will ultimately recognize a continuum of diseases that encompasses primary immunodeficiencies, autoimmune disorders, and autoinflammatory disorders, which might share subsets of genetic lesions.

Primary immunodeficiency diseases have played a main role in the development of novel therapeutic strategies. In 1968, SCIDX1 was the first disease to be cured by bone marrow transplantation (64), and in 2000 the same disease was the first true success of gene therapy. It is foreseeable that gene therapy will soon achieve further therapeutic successes in those immunodeficiencies where corrected cells have a strong selective advantage over unmodified populations. On the other hand, gene therapy involving molecules not providing such selective advantage will certainly be more challenging and will require more efficient targeting systems.
1. Fischer, A. 2001. Primary immunodeficiency diseases: an experimental model for molecular medicine. Lancet. 357:1863-1869.

2. Buckley, R.H. 2000. Primary immunodeficiency diseases due to defects in lymphocytes. N. Engl. J. Med. 343:1313-1324.

3. Noguchi, M., et al. 1993. Interleukin-2 receptor gamma chain mutation results in X-linked severe combined immunodeficiency in humans. Cell. 73:147-157.

4. Puel, A., Ziegler, S.F., Buckley, R.H., and Leonard, W.J. 1998. Defective IL7R expression in $\mathrm{T}(-) \mathrm{B}(+) \mathrm{NK}(+)$ severe combined immunodeficiency. Nat. Genet. 20:394-397.

5. Macchi, P., et al. 1995. Mutations of Jak-3 gene in patients with autosomal severe combined immune deficiency (SCID). Nature. 377:65-68.

6. Russell, S.M., et al. 1995. Mutation of Jak3 in a patient with SCID: essential role of Jak3 in lymphoid development. Science. 270:797-800.

7. Notarangelo, L.D., et al. 2001. Mutations in severe combined immune deficiency (SCID) due to JAK3 deficiency. Hum. Mutat. 18:255-263.

8. Chen, M., et al. 2000. Complex effects of naturally occurring mutations in the JAK3 pseudokinase domain: evidence for interactions between the kinase and pseudokinase domains. Mol. Cell. Biol. 20:947-956.

9. Zhou, Y.J., et al. 2001. Unexpected effects of FERM domain mutations on catalytic activity of Jak3: structural implication for Janus kinases. Mol. Cell. 8:959-969.

10. Sharfe, N., Shahar, M., and Roifman, C.M. 1997. An interleukin-2 receptor gamma chain mutation with normal thymus morphology. J. Clin. Invest. 100:3036-3043.

11. Schmalstieg, F.C., et al. 1995. Missense mutation in exon 7 of the common gamma chain gene causes a moderate form of X-linked combined immunodeficiency. J. Clin. Invest. 95:1169-1173.

12. Sharfe, N., Dadi, H.K., Shahar, M., and Roifman, C.M. 1997. Human immune disorder arising from mutation of the alpha chain of the interleukin-2 receptor. Proc. Natl. Acad. Sci. USA. 94:3168-3171.

13. Frucht, D.M., et al. 2001. Unexpected and variable phenotypes in a family with JAK3 deficiency. Genes Immun. 2:422-432.

14. Wildin, R.S., et al. 2001. X-linked neonatal diabetes mellitus, enteropathy and endocrinopathy syndrome is the human equivalent of mouse scurfy. Nat. Genet. 27:18-20.

15. 1997. An autoimmune disease, APECED, caused by mutations in a novel gene featuring two PHD-type zinc-finger domains. The Finnish-German APECED Consortium. Autoimmune Polyendocrinopathy-CandidiasisEctodermal Dystrophy. Nat. Genet. 17:399-403.

16. Nagamine, K., et al. 1997. Positional cloning of the APECED gene. Nat. Genet. 17:393-398.

17. Dupuis, S., et al. 2000. Human interferon-gamma-mediated immunity is a genetically controlled continuous trait that determines the outcome of mycobacterial invasion. Immunol. Rev. 178:129-137.

18. Dupuis, S., et al. 2001. Impairment of mycobacterial but not viral immunity by a germline human STAT1 mutation. Science. 293:300-303.

19. McDermott, M.F., et al. 1999. Germline mutations in the extracellular domains of the $55 \mathrm{kDa}$ TNF receptor, TNFR1, define a family of dominantly inherited autoinflammatory syndromes. Cell. 97:133-144.

20. Kastner, D.L., and O'Shea, J.J. 2001. A fever gene comes in from the cold. Nat. Genet. 29:241-242.

21. Rieux-Laucat, F., et al. 1995. Mutations in Fas associated with human lymphoproliferative syndrome and autoimmunity. Science. 268:1347-1349.

22. Fisher, G.H., et al. 1995. Dominant interfering Fas gene mutations impair apoptosis in a human autoimmune lymphoproliferative syndrome. Cell. 81:935-946.

23. Wang, J., et al. 1999. Inherited human Caspase 10 mutations underlie defective lymphocyte and dendritic cell apoptosis in autoimmune lymphoproliferative syndrome type II. Cell. 98:47-58.

24. Notarangelo, L.D., and Hayward, A.R. 2000. X-linked immunodeficiency with hyper-IgM (XHIM). Clin. Exp. Immunol. 120:399-405.

25. Ferrari, S., et al. 2001. Mutations of CD40 gene cause an autosomal recessive form of immunodeficiency with hyper IgM. Proc. Natl. Acad. Sci. USA. 98:12614-12619.

26. Doffinger, R., et al. 2001. X-linked anhidrotic ectodermal dysplasia with immunodeficiency is caused by impaired NF-kappaB signaling. Nat. Genet. 27:277-285.

27. Jain, A., et al. 2001. Specific missense mutations in NEMO result in hyper-IgM syndrome with hypohydrotic ectodermal dysplasia. Nat. Immunol. 2:223-228.

28. Revy, P., et al. 2000. Activation-induced cytidine deaminase (AID) deficiency causes the autosomal recessive form of the Hyper-IgM syndrome (HIGM2). Cell. 102:565-575

29. Villard, J., et al. 2001. MHC class II deficiency: a disease of gene regulation. Medicine (Baltimore). 80:405-418.

30. Schwarz, K., et al. 1996. RAG mutations in human B cell-negative SCID. Science. 274:97-99.

31. Villa, A., et al. 1998. Partial V(D)J recombination activity leads to Omenn syndrome. Cell. 93:885-896. 
32. Moshous, D., et al. 2001. ARTEMIS, a novel DNA double-strand break repair/V(D)J recombination protein, is mutated in human severe combined immune deficiency. Cell. 105:177-186.

33. Ma, Y., Pannicke, U., Schwarz, K., and Lieber, M.R. 2002. Hairpin opening and overhang processing by an ARTEMIS/DNA-dependent protein kinase complex in nonhomologous end joining and $\mathrm{V}(\mathrm{D}) \mathrm{J}$ recombination. Cell. 108:781-794.

34. O'Driscoll, M., et al. 2001. DNA ligase IV mutations identified in patients exhibiting developmental delay and immunodeficiency. Mol. Cell. 8:1175-1185.

35. Savitsky, K., et al. 1995. A single ataxia telangiectasia gene with a product similar to PI-3 kinase. Science. 268:1749-1753.

36. Hartley, K.O., et al. 1995. DNA-dependent protein kinase catalytic subunit: a relative of phosphatidylinositol 3-kinase and the ataxia telangiectasia gene product. Cell. 82:849-856.

37. Goldman, F.D., et al. 1998. Defective expression of p56lck in an infant with severe combined immunodeficiency. J. Clin. Invest. 102:421-429.

38. Elder, M.E., et al. 1994. Human severe combined immunodeficiency due to a defect in ZAP-70, a T cell tyrosine kinase. Science. 264:1596-1599.

39. Kung, C., et al. 2000. Mutations in the tyrosine phosphatase CD45 gene in a child with severe combined immunodeficiency disease. Nat. Med. 6:343-345.

40. Satterthwaite, A.B., and Witte, O.N. 2000. The role of Bruton's tyrosine kinase in B-cell development and function: a genetic perspective. Immunol. Rev. 175:120-127.

41. Minegishi, Y., et al. 1999. An essential role for BLNK in human B cell development. Science. 286:1954-1957.

42. Derry, J.M., Ochs, H.D., and Francke, U. 1994. Isolation of a novel gene mutated in Wiskott-Aldrich syndrome. Cell. 78:635-644.

43. Thrasher, A.J., Burns, S., Lorenzi, R., and Jones, G.E. 2000. The WiskottAldrich syndrome: disordered actin dynamics in haematopoietic cells. Immunol. Rev. 178:118-128.

44. Kim, A.S., Kakalis, L.T., Abdul-Manan, N., Liu, G.A., and Rosen, M.K. 2000. Autoinhibition and activation mechanisms of the Wiskott-Aldrich syndrome protein. Nature. 404:151-158.

45. Devriendt, K., et al. 2001. Constitutively activating mutation in WASP causes X-linked severe congenital neutropenia. Nat. Genet. 27:313-317.

46. Sturla, L., et al. 2001. Impairment of the Golgi GDP-L-fucose transport and unresponsiveness to fucose replacement therapy in LAD II patients. Pediatr. Res. 49:537-542.

47. Luhn, K., et al. 2001. The gene defective in leukocyte adhesion deficiency II encodes a putative GDP-fucose transporter. Nat. Genet. 28:69-72.

48. Sayos, J., et al. 1998. The X-linked lymphoproliferative-disease gene product SAP regulates signals induced through the co-receptor SLAM. Nature. 395:462-469.

49. Stepp, S.E., et al. 1999. Perforin gene defects in familial hemophagocytic lymphohistiocytosis. Science. 286:1957-1959.
50. Menasche, G., et al. 2000. Mutations in RAB27A cause Griscelli syndrome associated with haemophagocytic syndrome. Nat. Genet. 25:173-176.

51. Pastural, E., et al. 2000. Two genes are responsible for Griscelli syndrome at the same 15q21 locus. Genomics. 63:299-306.

52. Hirschhorn, R. 1999. Immunodeficiency disease due to deficiency of adenosine deaminase. In Primary immunodeficiency diseases: a molecular and genetic approach. H.D. Ochs, C.I.E. Smith, and J.M. Puck, editors. Oxford University Press. New York, New York, USA. 121-139.

53. Hershfield, M.S., and Mitchell, B.S. 2001. Immunodeficiency diseases caused by adenosine deaminase deficiency and purine nucleoside phosphorylase deficiency. In The metabolic and molecular bases of inherited disease. C.R. Scriver, A.L. Beaudet, W.S. Sly, and D. Valle, editors. McGraw-Hill. New York, New York, USA. 2585-2625.

54. Hirschhorn, R. 1996. Spontaneous in vivo reversion to normal of an inherited mutation in a patient with adenosine deaminase deficiency. Nat. Genet. 13:290-295.

55. Arredondo-Vega, F.X., et al. 2001. Adenosine deaminase deficiency with mosaicism for a "second-site suppressor" of a splicing mutation: decline in revertant $\mathrm{T}$ lymphocytes during enzyme replacement therapy. Blood. 99:1005-1013.

56. Stephan, V., et al. 1996. Atypical X-linked severe combined immunodeficiency due to possible spontaneous reversion of the genetic defect in T cells. N. Engl. J. Med. 335:1563-1567.

57. Ariga, T., et al. 2001. Spontaneous in vivo reversion of an inherited mutation in the Wiskott-Aldrich syndrome. J. Immunol. 166:5245-5249.

58. Wada, T., et al. 2001. Somatic mosaicism in Wiskott-Aldrich syndrome suggests in vivo reversion by a DNA slippage mechanism. Proc. Natl. Acad. Sci. USA. 98:8697-8702.

59. Cavazzana-Calvo, M., et al. 2000. Gene therapy of human severe combined immunodeficiency (SCID)-X1 disease. Science. 288:669-672.

60. Bordignon, C., et al. 1995. Gene therapy in peripheral blood lymphocytes and bone marrow for ADA-immunodeficient patients. Science. 270:470-475.

61. Kohn, D.B., et al. 1998. T lymphocytes with a normal ADA gene accumulate after transplantation of transduced autologous umbilical cord blood CD34+ cells in ADA-deficient SCID neonates. Nat. Med. 4:775-780.

62. Malech, H.L., et al. 1997. Prolonged production of NADPH oxidasecorrected granulocytes after gene therapy of chronic granulomatous disease. Proc. Natl. Acad. Sci. USA. 94:12133-12138.

63. Bauer, T.R., and Hickstein, D.D. 2000. Gene therapy for leukocyte adhesion deficiency. Curr. Opin. Mol. Ther. 2:383-388.

64. Gatti, R.A., Meuwissen, H.J., Allen, H.D., Hong, R., and Good, R.A. 1968. Immunological reconstitution of sex-linked lymphopenic immunological deficiency. Lancet. 2:1366-1369. 